\title{
Underwater endoscopic mucosal resection for anal canal neoplasia in a patient with human immunodeficiency virus infection
}

\author{
Mami Hirai, Yasushi Yamasaki, Keita Harada, Hiroyuki Okada \\ Okayama University Hospital, Okayama, Japan
}

Human immunodeficiency virus (HIV)-positive patients are at high risk of anal cancer because the majority are concurrently infected with human papillomavirus (HPV) in the anus [1]. As high-grade anal intraepithelial neoplasia (HAIN), precursor of cancer, is caused by HPV infection, early detection and treatment of HAIN is recommended [2].

Despite an early diagnosis of HAIN, conventional endoscopic treatment is often difficult, because of the narrow lumen, poor operability and susceptibility to bleeding during the procedure. Furthermore, when a large bleb arises in the anal canal as a result of submucosal injection, it sometimes makes endoscopic resection more difficult and leads to incomplete resection. Theoretically, the novel technique of underwater endoscopic mucosal resection (UEMR), which does not require submucosal injection, can overcome these technical difficulties [3]. Herein we report how we applied UEMR for an anal canal lesion.

An HIV-positive man in his 60s underwent screening colonoscopy, during which a $10-\mathrm{mm}$ elevated lesion was detected in the anal canal (Fig. 1A). As biopsies showed HAIN, we performed UEMR. After air deflation in the rectum and anus, the lumen was filled with physiological saline and the lesion was adequately visualized. The lesion was easily captured by a polypectomy snare $(15 \mathrm{~mm}$, Captivator II; Boston Scientific, USA), and removed en bloc with an electric current (Fig. 1B). The submucosal vessels remained in the wound without bleeding (Fig. 1C) and were coagulated safely (Fig. 1D). Histological examination revealed HAIN with positive staining for P16, a marker of HPV infection (Fig. 2A). There was no recurrence at follow up 4 months later (Fig. 2B). UEMR was effective in treating this anal canal lesion.

\section{Acknowledgment}

We are sincerely grateful to the medical staff members in our endoscopy unit for their collaboration on this work

Department of Gastroenterology, Okayama University Hospital, Okayama, Japan

Conflict of Interest: None

Correspondence to: Yasushi Yamasaki, Department of Gastroenterology, Okayama University Hospital, 2-5-1 Shikata-cho, Kita-ku, Okayama-city, Okayama 700-8558, Japan,

e-mail: yasshifive@yahoo.co.jp

Received 20 March 2018; accepted 27 March 2018; published online 4 June 2018

DOI: https://doi.org/10.20524/aog.2018.0282

๑) 2018 Hellenic Society of Gastroenterology

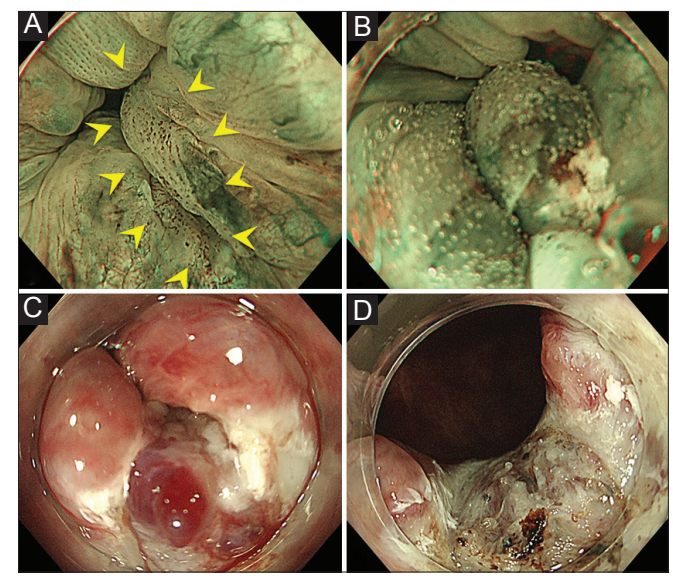

Figure 1 Underwater endoscopic mucosal resection for anal canal neoplasia in a patient with human immunodeficiency virus infection. (A) An elevated lesion $10 \mathrm{~mm}$ in size was detected in the anal canal. (B) The lesion was captured by a polypectomy snare. (C) The lesion was completely removed by underwater endoscopic mucosal resection without bleeding. (D) Submucosal vessels were coagulated and complete resection was achieved

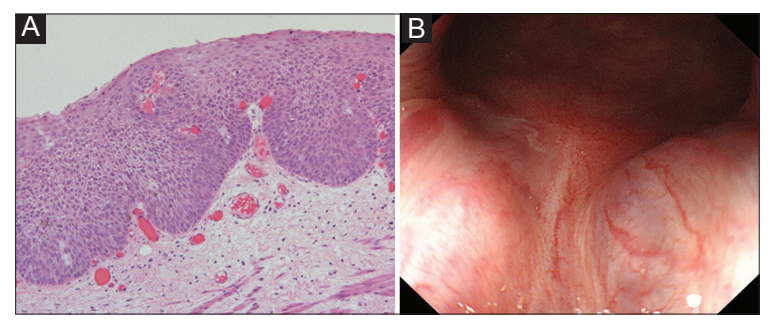

Figure 2 Histopathological examination and follow-up endoscopy. (A) On histopathological examination, nuclear atypia was found up to the upper third of the mucosa. Hematoxylin and eosin stain $(\times 40)$. (B) There was no recurrence 4 months after treatment

Video 1 Underwater endoscopic mucosal resection for the anal canal lesion. After air deflation, normal saline was infused. Marking was performed by the tip of the snare. The lesion was then captured and transected while underwater. Finally, the remaining vessels were coagulated

\section{References}

1. D'Souza G, Wiley DJ, Li X, et al. Incidence and epidemiology of anal cancer in the Multicenter AIDS Cohort Study (MACS). J Acquir Immune Defic Syndr 2008;48:491-499.

2. Scholefield JH, Castle MT, Watson NFS. Malignant transformation of high-grade anal intraepithelial neoplasia. BrJ Surg 2005;92:1133-1136.

3. Binmoeller KF, Weilert F, Shah J, Bhat Y, Kane S. "Underwater" EMR without submucosal injection for large sessile colorectal polyps (with video). Gastrointest Endosc 2012;75:1086-1091. 